\title{
Oxysterols Modulate the Acute Effects of Ethanol on Hippocampal N-Methyl-D-Aspartate Receptors, Long-Term Potentiation, and Learning
}

\author{
(D) Yukitoshi Izumi, Steven J. Mennerick, James J. Doherty, and Charles F. Zorumski \\ Department of Psychiatry and Taylor Family Institute for Innovative Psychiatric Research, Washington University School of \\ Medicine, St. Louis, Missouri (Y.I., S.J.M., C.F.Z.); and Sage Therapeutics, Cambridge, Massachusetts (J.J.D.)
}

Received October 13, 2020; accepted January 8, 2021

\begin{abstract}
Ethanol is a noncompetitive inhibitor of $N$-methyl-D-aspartate receptors (NMDARs) and acutely disrupts hippocampal synaptic plasticity and learning. In the present study, we examined the effects of oxysterol positive allosteric modulators (PAMs) of NMDARs on ethanol-mediated inhibition of NMDARs, block of long-term potentiation (LTP) and longterm depression (LTD) in rat hippocampal slices, and defects in one-trial learning in vivo. We found that 24S-hydroxycholesterol and a synthetic oxysterol analog, SGE-301, overcame effects of ethanol on NMDAR-mediated synaptic responses in the CA1 region but did not alter acute effects of ethanol on LTD; the synthetic oxysterol, however, overcame acute inhibition of LTP. In addition, both oxysterols overcame persistent effects of ethanol on LTP in vitro, and the synthetic analog reversed
\end{abstract}

defects in one-trial inhibitory avoidance learning in vivo. These results indicate that effects of ethanol on both LTP and LTD arise by complex mechanisms beyond NMDAR antagonism and that oxysterol NMDAR PAMS may represent a novel approach for preventing and reversing acute ethanol-mediated changes in cognition.

\section{SIGNIFICANCE STATEMENT}

Ethanol acutely inhibits hippocampal NMDARs, LTP, and learning. This study found that certain oxysterols that are NMDARpositive allosteric modulators can overcome the acute effects of ethanol on NMDARs, LTP, and learning. Oxysterols differ in their effects from agents that inhibit integrated cellular stress responses.

\section{Introduction}

Ethanol can acutely alter memory (Oslin and Cary, 2003; White, 2003; Abrahao et al., 2017) and impair long-term synaptic plasticity involved in memory formation (Izumi et al., 2005b; Zorumski et al., 2014). Ethanol is a noncompetitive inhibitor of $N$-methyl-D-aspartate receptors (NMDARs) that contribute to synaptic plasticity but is only a partial antagonist at concentrations that block synaptic plasticity. It is thus uncertain whether effects on NMDARs drive ethanolinduced impairments. Some effects of ethanol on synaptic plasticity, particularly inhibition of long-term potentiation (LTP), involve a form of metaplasticity resulting from activation of NMDARs that remain unblocked by ethanol during

Work in the authors' laboratory is supported by National Institutes of Health National Institute of Mental Health [Grant MH101874] and MH114866], National Institute on Alcohol Abuse and Alcoholism [Grant AA026753], the Taylor Family Institute for Innovative Psychiatric Research, and the Bantly Foundation.

C.F.Z is a member of the Scientific Advisory Board of Sage Therapeutics. J.J.D. is employed by Sage Therapeutics. Sage Therapeutics did not fund this research. Y.I. and S.J.M. have no conflicts of interest to disclose. There are no other competing financial interests.

https://doi.org/10.1124/jpet.120.000376. acute drug exposures (Izumi et al., 2005b; Tokuda et al., 2011). This NMDAR-dependent LTP inhibition involves complex signaling (Zorumski and Izumi, 2012), including activation of the cellular integrated stress response (ISR), a mechanism that regulates neuronal plasticity and memory under pathologic conditions (Izumi and Zorumski, 2020). Ethanol activates ISR (Chen et al., 2008; Magne et al., 2011), and agents that block ISR overcome the effects of ethanol on LTP, longterm depression (LTD), and a form of hippocampal-dependent, one-trial inhibitory avoidance learning (Izumi and Zorumski, 2020). These observations suggest that modulation of stress responses may effectively prevent or reverse detrimental effects of ethanol on cognition, particularly learning defects associated with acute alcohol-induced memory blackouts (White, 2003)

One of the agents that overcomes the acute effects of ethanol on synaptic plasticity and learning is an agonist at liver X receptors (LXRs) (Izumi and Zorumski, 2020). The test of the LXR agonist (GW3965) was prompted by work showing that LXR activation dampens ISR by modulating plasma membrane composition (Rong et al., 2013). Effects of GW3965 suggest that other agents that modulate LXRs may also have beneficial effects on ethanol's synaptic and cognitive effects.

ABBREVIATIONS: ACSF, artificial cerebrospinal fluid; CDX, 2-hydroxypropyl- $\beta$-cyclodextrin; EPSP, excitatory postsynaptic potential; ER, endoplasmic reticulum; fEPSP, field excitatory postsynaptic potential; HFS, high-frequency stimulus; IO, input-output; ISR, integrated stress response; LFS, low-frequency stimulation; LTD, long-term depression; LTP, long-term potentiation; LXR, liver X receptor; NMDA, N-methylD-aspartate; NMDAR, N-methyl-D-aspartate receptor; PAM, positive allosteric modulator; PCP, phencyclidine; 24S-HC, 24S-hydroxycholesterol. 
Certain side-chain-oxidized derivatives of cholesterol (oxysterols) are agonists for LXRs (Russell et al., 2009; Sun et al., $2016 \mathrm{~b})$. A prototypic example is $24 \mathrm{~S}-$ hydroxycholesterol (24S$\mathrm{HC}$ ), the major cholesterol metabolite in the brain (Janowski et al., 1999). 24S-HC is an endogenous neuromodulator that, in addition to effects on LXR, is a positive allosteric modulator (PAM) of NMDARs that also promotes hippocampal plasticity (Paul et al., 2013; Linsenbardt et al., 2014). Furthermore, synthetic derivatives of 24S-HC (Paul et al., 2013) have shown promise as potential therapeutic agents for treating cognitive dysfunction in certain neuropsychiatric illnesses (Koenig et al., 2019). For these reasons, we were interested in determining whether $24 \mathrm{~S}-\mathrm{HC}$ and a synthetic derivative, SGE301 , would alter acute effects of ethanol on hippocampal function and learning. We chose SGE-301 for these studies because it mimics the effects of 24S-HC on NMDARs and has good brain bioavailability after systemic administration (Paul et al., 2013).

\section{Materials and Methods}

Chemicals. SGE-301 was a gift from Sage Therapeutics (Cambridge, MA) (Paul et al., 2013). Other chemicals were purchased from Sigma Chemical Company (St. Louis, MO). For ex vivo slice experiments, SGE-301 or 24S-HC was dissolved in DMSO and administered at concentrations noted in the text. The concentration of DMSO was typically $0.1 \%$ and has no effect on hippocampal responses or synaptic plasticity (Izumi and Zorumski, 2020; Popiolek et al., 2020). For in vivo experiments, $5 \mathrm{mg}$ SGE-301 was dissolved in $3.3 \mathrm{ml}$ of 2-hydroxypropyl- $\beta$-cyclodextrin (CDX) (catalog number 332607; Sigma) (1.5 g $\mathrm{CDX}+3 \mathrm{ml}$ water) and administered at a final dose of $3 \mathrm{mg} / \mathrm{kg}$ i.p. This dose of SGE-301 was selected based on prior behavioral studies we have done with this agent (Paul et al., 2013).

Hippocampal Slice Preparation. The Washington University Institutional Animal Care and Use Committee approved all protocols for animal experiments according to NIH guidelines. Using methods described previously, hippocampal slices were prepared from postnatal day 28-32 Harlan (Indianapolis, IN) Sprague-Dawley male albino rats (Tokuda et al., 2010, 2011). For slice preparation, rats were anesthetized with isoflurane, and dissected hippocampi were pinned at their ventral pole on an agar base in ice-cold artificial cerebrospinal fluid (ACSF) that contained (in millimolars) $124 \mathrm{NaCl}, 5 \mathrm{KCl}, 2$ $\mathrm{MgSO}_{4}, 2 \mathrm{CaCl}_{2}, 1.25 \mathrm{NaH}_{2} \mathrm{PO}_{4}, 22 \mathrm{NaHCO}_{3}$, and 10 glucose, bubbled with $95 \% \mathrm{O}_{2}$ and $5 \% \mathrm{CO}_{2}$ at $4-6^{\circ} \mathrm{C}$. The dorsal two-thirds of the hippocampus was cut into $500-\mu \mathrm{m}$ slices using a rotary tissue slicer and kept in a chamber with gassed ACSF at $30^{\circ} \mathrm{C}$ for at least 1 hour before use in physiology studies.

Hippocampal Slice Physiology. Slices were transferred one at a time to a submersion-recording chamber at $30^{\circ} \mathrm{C}$ and perfused with the ACSF described above at $2 \mathrm{ml} / \mathrm{min}$. We did not include inhibitors of $\mathrm{GABA}_{\mathrm{A}}$ receptors in our recording solutions to avoid nonphysiologic network effects resulting from disinhibition (Izumi et al., 2005a, 2007; Tokuda et al., 2011; Izumi and Zorumski, 2020). Extracellular recordings were obtained from the apical dendritic layer (stratum radiatum) of area CA1 to monitor field excitatory postsynaptic potentials (fEPSPs)

fEPSPs in the stratum radiatum of CA1 were evoked once per minute with 0.1-millisecond constant-current pulses administered to the Schaffer collateral pathway via a bipolar stimulating electrode. Stimulus intensity was set to half-maximal based on a baseline inputoutput (IO) curve. LTP was induced using a $100 \mathrm{~Hz} \times 1$ second highfrequency stimulus (HFS) at the same intensity. LTD was induced with low-frequency stimulation (LFS) consisting of 900 single pulses at $1 \mathrm{~Hz}$. IO curves were repeated 60 minutes after HFS or LFS and were the primary outcome measure of synaptic change by comparison with baseline IO curves.
For experiments examining isolated NMDAR-mediated fEPSPs, dendritic responses were recorded in stratum radiatum at low frequency $(1 / \mathrm{min})$ in ACSF containing $0.1 \mathrm{mM} \mathrm{Mg}^{2+}$ and $2.5 \mathrm{mM}$ $\mathrm{Ca}^{2+}$ to promote NMDAR activation and $30 \mu \mathrm{M}$ 6-cyano-7-nitroquinoxaline-2,3-dione to block AMPARs (Izumi et al., 2005b). fEPSPs under these conditions are completely inhibited by the NMDAR antagonist 2-amino-5-phosphonovalerate (Izumi et al., 2005b, 2006). We quantified NMDAR fEPSPs by the rising slope of the field potential (12-24 points) before and at the end of drug treatment, and effects of drugs were based on comparison of the $50 \%$ maximal point on IO curves before and after drug exposure.

Behavioral Studies. To assess in vivo memory formation, P28-32 rats were tested in a one-trial inhibitory avoidance learning task that has been associated with CA1 hippocampal LTP (Whitlock et al., 2006; Tokuda et al., 2010). The testing apparatus has two chambers, one of which is illuminated, whereas the second chamber is kept dark. These chambers have a floor of stainless steel rods (4 mm diameter, spaced $10 \mathrm{~mm}$ apart), through which an electrical shock can be delivered in the dark chamber $(12 \times 20 \times 16 \mathrm{~cm})$. The lit compartment $(30 \times 20 \times$ $16 \mathrm{~cm}$ ) was illuminated by four 13-W lights, and light intensity in the lit chamber was 1000 lux, whereas the dark chamber was $<10$ lux.

At the time of study, rats were initially placed in the lit chamber and allowed to move freely between the chambers for 10 minutes. Subsequently, animals were administered SGE-301 (3 mg/kg i.p.) or vehicle 2 hours prior to ethanol or saline administration. At 15 minutes prior to being placed back in the apparatus, rats were treated with either saline or ethanol ( $2 \mathrm{~g} / \mathrm{kg}$ i.p.). This dose of ethanol results in acute blood and cerebrospinal fluid levels of ethanol near $20 \mathrm{mM}$ 15 minutes after injection (Izumi and Zorumski, 2020). Rats were then placed in the lit compartment and allowed to explore the apparatus for up to 300 seconds. Upon complete entry into the dark chamber, rats were immediately given a foot shock. Animals were then removed from the apparatus and returned to their home cages. On the next day of testing, rats were placed in the lit chamber without drug treatment, and the latency to enter the dark compartment was recorded over a 300 -second trial.

Experimental Design and Statistical Analysis. Data were collected and analyzed using PClamp (Molecular Devices, Union City, CA) and are expressed as means \pm S.E.M. At 60 minutes after HFS or LFS, fEPSPs were normalized to baseline recordings (taken as 100\%). A two-tailed Student's $t$ test was used for all comparisons of fEPSPs between groups. When appropriate (Fig. 1), paired $t$ tests were used. Statistical comparisons were based on analysis of IO curves at baseline and 60 minutes after HFS or LFS to determine the degree of change in the maximal rising slope of fEPSPs evoked by stimuli at the $50 \%$ point on the IO curves, with $P<0.05$ considered significant (Izumi and Zorumski, 2020). For behavioral studies in Fig. 4, data were analyzed by one-way analysis of variance followed by Dunnett's multiple comparisons test. Numbers reported in the text for statistical analyses are the number $(N)$ of animals studied in a given condition. Statistics were performed using commercial software [SigmaStat (Systat Software, Inc., Richmond City, CA) or GraphPad Prism version 8.3.0 for Windows (www.graphpad.com; GraphPad Software, San Diego, CA)]. Data in figures showing fEPSPs display continuous monitoring of responses at low frequency and thus may differ from numerical results described in the text, which are based on analyses of IO curves.

\section{Results}

Previously, we found that $60 \mathrm{mM}$ ethanol acutely and reversibly inhibits isolated NMDAR-mediated fEPSPs in the CA1 region of rat hippocampal slices by about $50 \%$, largely via inhibition of a subtype of NMDARs expressing GluN2B subunits (Izumi et al., 2005b). In initial experiments, we examined whether an oxysterol PAM of NMDARs alters the effects of ethanol on synaptic responses mediated by these 

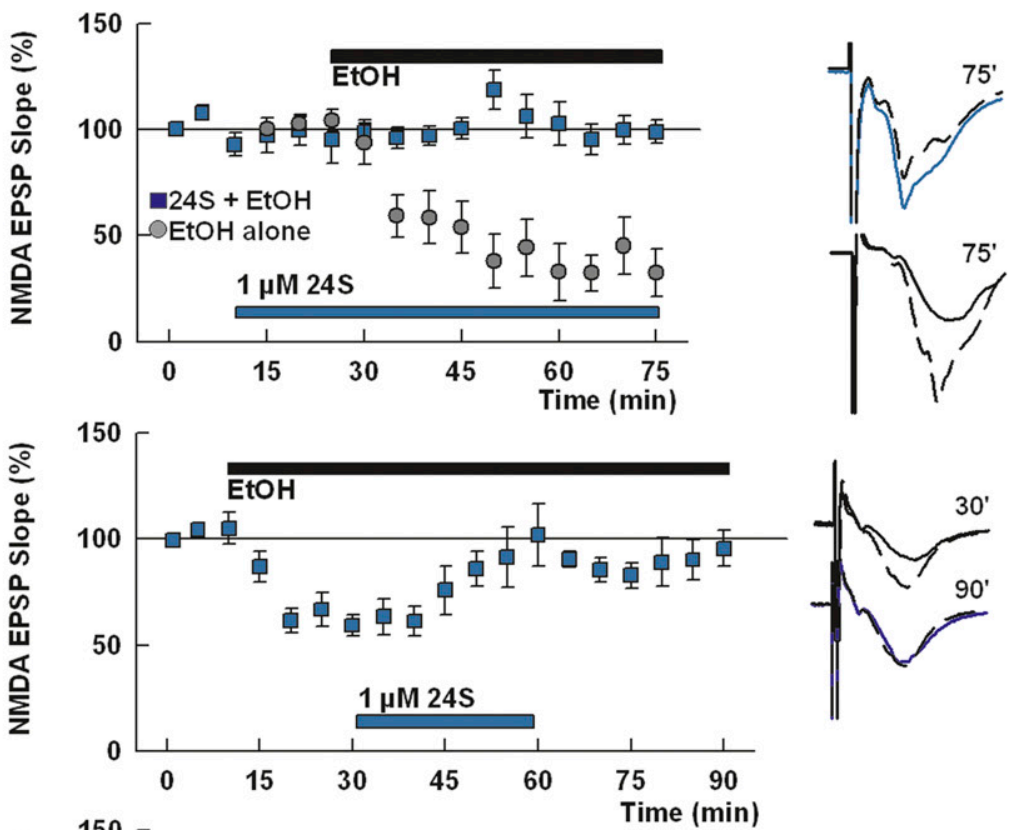

Fig. 1. 24S-HC and a synthetic oxysterol analog prevent effects of ethanol on NMDA EPSPs. (A) The graph shows the time course of change of isolated NMDAR-mediated fEPSPs in the presence of $1 \mu \mathrm{M} 24 \mathrm{~S}-\mathrm{HC}$ (blue bar, denoted as $24 \mathrm{~S}$ in the graph) followed by $60 \mathrm{mM}$ ethanol (denoted as EtOH in the graph) normalized to baseline (100\%). In the absence of 24S-HC, ethanol depressed NMDAR-mediated fEPSPs. (B) Even when administered after $60 \mathrm{mM}$ ethanol, which acutely inhibits NMDAR responses, $24 \mathrm{~S}-\mathrm{HC}$ dampened the block of NMDA fEPSPs. (C) SGE-301 (red bar), a synthetic mimic of 24S-HC, also prevents effects of ethanol on NMDA fEPSPs. (D) Akin to 24S-HC, SGE-301 reverses the effects of ethanol on NMDA fEPSPs when administered after block is established. Traces to the right of the graphs show representative NMDA fEPSPs at the times denoted, with baseline responses shown as dashed lines. Calibration: $1 \mathrm{mV}, 5$ milliseconds.

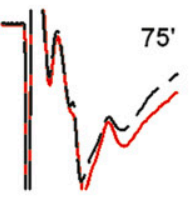

receptors. 24S-HC is an endogenous oxysterol, the major metabolite of cholesterol in brain (Sun et al., 2016a,b), and an NMDAR PAM that enhances CA1 NMDAR fEPSPs at concentrations in the low micromolar range (Paul et al., 2013; Linsenbardt et al., 2014). At a threshold concentration of $1 \mu \mathrm{M}$ in hippocampal slices, 24S-HC prevented the ability of $60 \mathrm{mM}$ ethanol to inhibit NMDAR fEPSPs when administered prior to and during ethanol exposure $(96.2 \% \pm 6.3 \%$ of baseline responses vs. $39.5 \% \pm 10.1 \%$ of baseline in the presence of ethanol alone, $P=0.0004 ; N=5$; Fig. $1 \mathrm{~A}$ ). At this concentration, 24S-HC had little direct effect on NMDAR responses (Fig. 1A). 24S-HC also reversed the effects of ethanol on NMDAR-mediated fEPSPs when administered after ethanolinduced NMDAR inhibition and in the continued presence of ethanol $(95.6 \% \pm 8.5 \%$ of baseline after $24 \mathrm{~S}-\mathrm{HC}$ administration vs. $53.9 \% \pm 5.5 \%$ with ethanol alone, $N=5, P=0.03137$ by paired $t$ test vs. ethanol alone; Fig. 1B). Similarly, $1 \mu \mathrm{M}$
SGE-301, a synthetic mimic of 24S-HC (Paul et al., 2013), prevented the effects of ethanol on NMDA fEPSPs and had no clear effect on these responses on its own at this concentration in slices $(104.9 \% \pm 5.0 \%, P=0.0014$ vs. ethanol alone; Fig. $1 \mathrm{C}$; $N=5$ ) (Sun et al., 2016a). SGE-301 also overcame the effects of ethanol on NMDAR fEPSPs when administered after inhibition by ethanol $(55.9 \% \pm 6.5 \%$ in the presence of ethanol alone vs. $106.9 \% \pm 4.4 \%$ with ethanol + SGE-301, $N=5$, $P<0.0001$ by paired test; Fig. 1D).

In prior studies (Izumi et al., 2005b), we found that $60 \mathrm{mM}$ ethanol acutely but reversibly inhibits homosynaptic LTD induced by $1 \mathrm{~Hz} \times 900$ pulse LFS of the Schaffer collateral pathway and that ethanol-mediated LTD inhibition mimics the effects of a selective antagonist of GluN1/GluN2B-expressing NMDARs. We thus examined whether $24 \mathrm{~S}-\mathrm{HC}$ altered the effects of ethanol on CA1 LTD. In naïve slices, LFS induced robust homosynaptic LTD (fEPSP slope: $53.9 \% \pm 5.5 \%$ of 
baseline 60 minutes after LFS; $N=6$, Fig. 2A). Surprisingly, despite overcoming the effects of ethanol on NMDAR fEPSPs (Fig. 1, A and B), 24S-HC had no effect on the ability of ethanol to block LTD (fEPSP slope: $99.3 \% \pm 3.5 \%$ of baseline 60 minutes after LFS, $N=5$ vs. $98.7 \% \pm 3.8 \%$ of baseline with ethanol alone, $N=6, P=0.3796 ; P<0.0001$ for each vs. control LTD; Fig. 2A). 24S-HC alone does not inhibit LTD (Popiolek et al., 2020). Similarly, SGE-301 failed to overcome the effects of ethanol on LTD $(104.2 \% \pm 6.0 \%$ of baseline, $N=$ 5; $P=0.4430$ vs. ethanol alone; $P=0.0002$ vs. control LTD; Fig. 2B). Neither $24 \mathrm{~S}-\mathrm{HC}(52.3 \% \pm 5.2 \%$ of baseline, $N=5 ; P=$ 0.8396 vs. control LTD) nor SGE-301 $(60.6 \% \pm 4.6 \%$ of baseline, $N=5 ; P=0.3866$ ) had any effect on LTD when administered alone.

We previously found that 24S-HC promotes LTP (Paul et al., 2013). Thus, given effects on the block of NMDA EPSPs, we next examined the effects of $24 \mathrm{~S}-\mathrm{HC}$ on acute inhibition of LTP by $60 \mathrm{mM}$ ethanol. We found that $24 \mathrm{~S}-\mathrm{HC}$ failed to alter the ability of ethanol to block LTP induced by $100 \mathrm{~Hz} \times 1$ second HFS at either 1 or $10 \mu \mathrm{M}$ [ $1 \mu \mathrm{M}$ 24S-HC: $95.5 \% \pm$ $7.2 \%$ of baseline fEPSPs 60 minutes after HFS (white circles);
$10 \mu \mathrm{M}: 94.3 \% \pm 9.8 \%$ of baseline, $N=5$ and 6 vs. ethanol alone: $96.1 \% \pm 3.2 \%, N=6, P=0.9371$ and 0.8649 , respectively; Fig. 2C]. In control slices, HFS readily induced LTP [black triangles, $148.4 \% \pm 6.9 \%$ of baseline 60 minutes after HFS, $N=6 ; P=0.0001$ vs. ethanol alone (black circles); $P=0.0005$ vs. ethanol $+1 \mu \mathrm{M} 24 \mathrm{~S}-\mathrm{HC}$ and $P=0.0011$ vs. ethanol $+10 \mu \mathrm{M}$ 24S-HC; Fig. 2C]. 24S-HC alone did not alter LTP in naïve slices at either $1 \mu \mathrm{M}(130.0 \% \pm 4.6 \%$ of baseline, $N=5 ; P=$ 0.0632 vs. control LTP; Fig. $2 \mathrm{C})$ or $10 \mu \mathrm{M}(139.5 \% \pm 5.7 \%$ of baseline, $N=5 ; P=0.3587$ vs. control LTP; not shown). In contrast to what we observed with $24 \mathrm{~S}-\mathrm{HC}$, we found that SGE-301 reversed the acute effects of ethanol on LTP $(155.6 \% \pm 16.1 \%$ of baseline, $N=5 ; P=0.0032$ vs. ethanol alone and $P=0.6712$ vs. control LTP; Fig. 2D). When administered alone, SGE-301 had no effect on LTP induced by a single $100 \mathrm{~Hz} \times 1$ second HFS $(154.7 \% \pm 15.0 \%$ of baseline, $N=5 ; P=0.6947$ ).

As noted earlier, ethanol's block of LTP involves a form of negative metaplasticity (Zorumski and Izumi, 2012) dependent upon untimely activation of NMDARs prior to delivery of HFS; this defect in LTP induction persists for over an hour

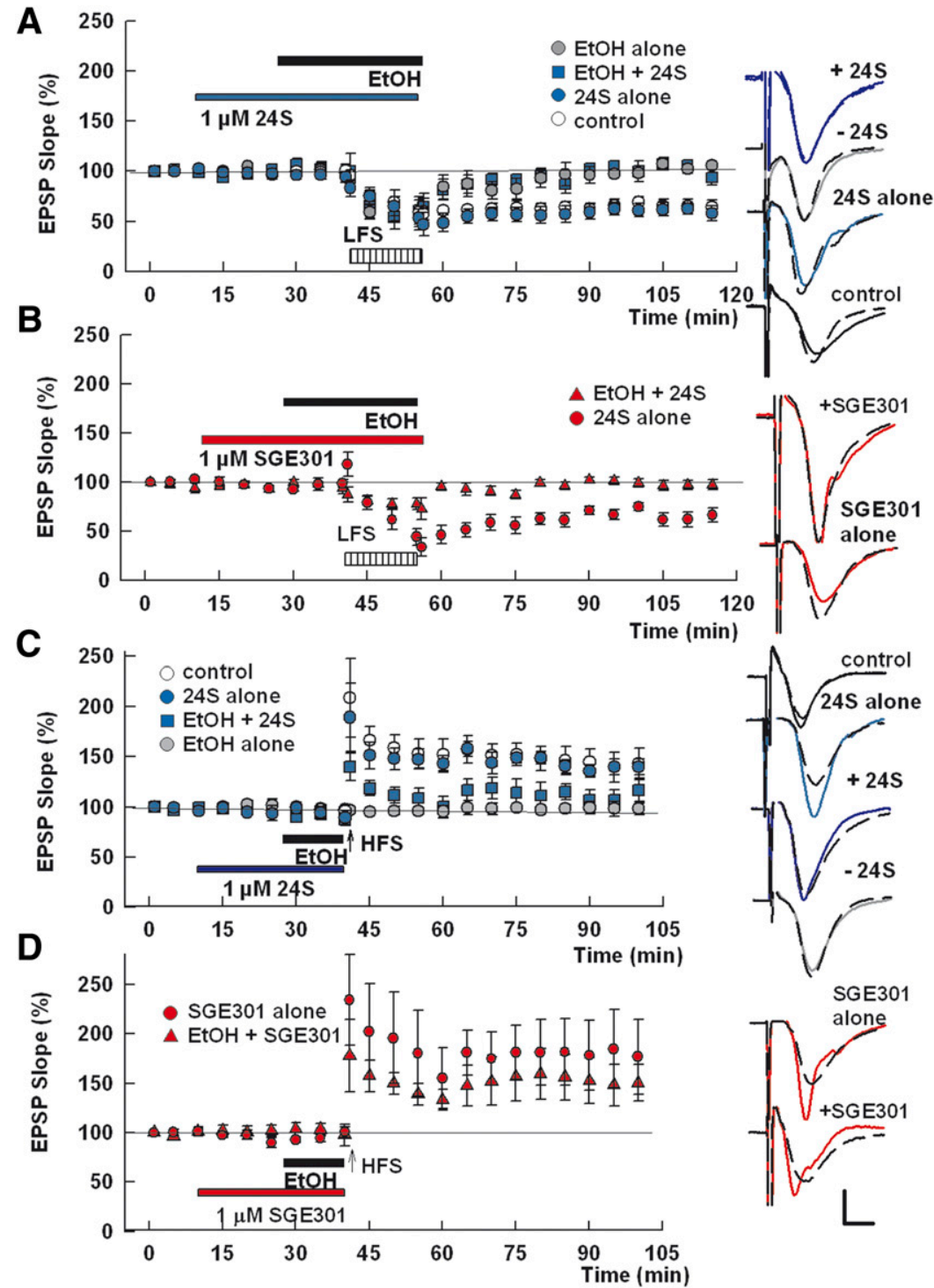

Fig. 2. Disparate interaction of $24 \mathrm{~S}-\mathrm{HC}$ and SGE-301 with the acute effects of ethanol on long-term synaptic plasticity. (A) Despite altering effects of ethanol on NMDA EPSPs, 24S-HC (blue bar) did not alter the block of homosynaptic LTD of AMPAR fEPSPs by $60 \mathrm{mM}$ ethanol. Administration of 1-Hz LFS (hatched bar) induces LTD in control naïve slices and in slices treated with $24 \mathrm{~S}-\mathrm{HC}$. Gray circles show the effects of ethanol alone, whereas blue squares depict the effects of ethanol plus 24S-HC. (B) Similarly, SGE-301 failed to alter acute LTD inhibition by ethanol. LTD is induced in the presence of SGE-301 alone. (C) Akin to effects on LTD, $1 \mu \mathrm{M} 24 \mathrm{~S}-\mathrm{HC}$ did not alter the acute effects of ethanol on LTP. The 100-Hz HFS (arrow) induces LTP in control naïve slices and in slices treated with $1 \mu \mathrm{M} 24 \mathrm{~S}-\mathrm{HC}$. Ethanol inhibits LTP induction in the presence or absence of 24S-HC. (D) Unlike 24S-HC, SGE-301 prevented acute LTP inhibition by ethanol. Administration of 24S-HC alone did not alter LTP induction. Traces to the right of the graphs show representative fEPSPs 60 minutes after the conditioning stimulation (solid lines), with dashed lines as control baseline responses. Delivery of $100 \mathrm{~Hz} \times 1$ second HFS is denoted by the arrows in (C and D). Calibration: $1 \mathrm{mV}, 5$ milliseconds. 
after ethanol washout (Izumi et al., 2005b; Tokuda et al., 2011; Zorumski et al., 2014). Thus, we also examined whether $24 \mathrm{~S}$ $\mathrm{HC}$ altered the persisting effects of ethanol. In contrast to acute LTP inhibition by ethanol, the prolonged effects of ethanol on LTP were eliminated in the presence of $24 \mathrm{~S}-\mathrm{HC}$ (fEPSP slopes: $98.1 \% \pm 6.5 \%$ of baseline 60 minutes after HFS following ethanol alone vs. $139.2 \% \pm 7.6 \%$ in the presence of 24S-HC, $N=6$ and 5, respectively; $P=0.0025$ by two-tailed Student's $t$ test; Fig. 3A). We also found that $24 \mathrm{~S}-\mathrm{HC}$ overcame the persisting effects of ethanol when administered after ethanol exposure $(164.7 \% \pm 14.7 \%$ of baseline, $N=5 ; P=$ 0.0017 vs. ethanol alone; Fig. 3B). Similar to $24 \mathrm{~S}-\mathrm{HC}$, SGE301 overcame persistent LTP inhibition by ethanol $(153.6 \% \pm$ $13.4 \%$ of baseline, $N=5 ; P=0.0034$ vs. ethanol; Fig. $3 \mathrm{C}$ ).

To determine whether the interaction between oxysterols and ethanol on synaptic plasticity predicts behavioral effects of ethanol, we examined whether oxysterols alter cognitive impairment by ethanol in vivo. For these studies, we used a one-trial inhibitory avoidance learning task that has previously been linked to hippocampal LTP (Whitlock et al., 2006; Tokuda et al., 2010) and that we recently showed is altered by acute ethanol administration (Izumi and Zorumski, 2020). When administered at $2 \mathrm{~g} / \mathrm{kg}$ i.p. 15 minutes prior to training, ethanol markedly reduced learning in the one-trial task when rats were tested 24 hours after training (Fig. 4). Based on our observations with the oxysterols (Figs. 1-3), we examined whether the 24S-HC analog SGE-301 altered these amnesic effects of ethanol. We used SGE-301 for these experiments because of its brain bioavailability after systemic administration (Paul et al., 2013) along with consistent effects on LTP in the presence of ethanol (Figs. 2 and 3). Rats treated with CDX, the vehicle used for in vivo administration of SGE-301, prior to initial exposure to the test chamber learned the task readily and remained in the lit chamber for $260.0 \pm 40.0$ seconds $(N=$ 5) 24 hours after initial exposure and shock. Animals treated with ethanol plus CDX (vehicle for SGE-301) prior to initial exposure failed to learn the task and entered the dark chamber in $6.0 \pm 2.0$ seconds $(N=5)$ upon exposure to the apparatus 24 hours later $(P<0.0003$ vs. CDX controls). When administered at $3 \mathrm{mg} / \mathrm{kg}$ i.p. 2 hours prior to ethanol, SGE-301 markedly diminished the ethanol-mediated learning defect (latency to enter the dark chamber: $253.0 \pm 32.0$ seconds, $N=$ $5 ; P<0.0003$ vs. vehicle + ethanol-treated controls); this duration in the lit chamber did not differ from controls $(P=$ 0.8947; Fig. 4). SGE-301 alone had no effect on learning the task (267.6 \pm 32.4 seconds, $N=5, P=0.8863$ vs. CDX-alone controls; Fig. 4).

\section{Discussion}

We recently found that the LXR agonist GW3965, an agent that affects sterol metabolism (Wang and Tontonoz, 2018) and inhibits endoplasmic reticulum (ER) stress (Rong et al., 2013), potently and effectively overcomes the acute effects of ethanol

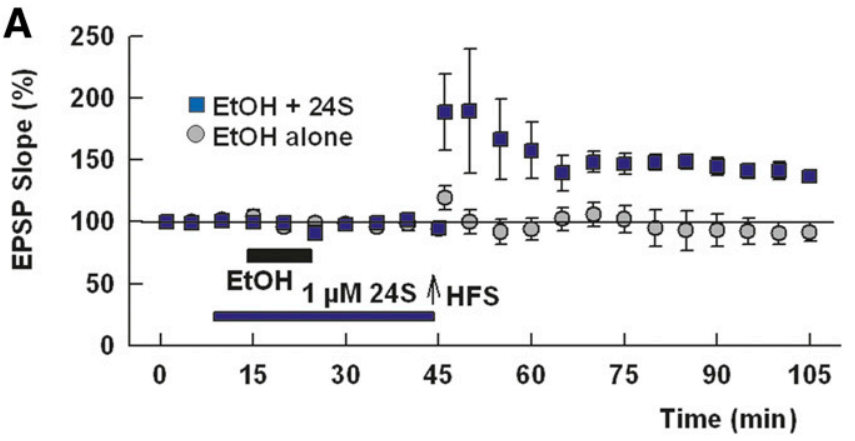

B
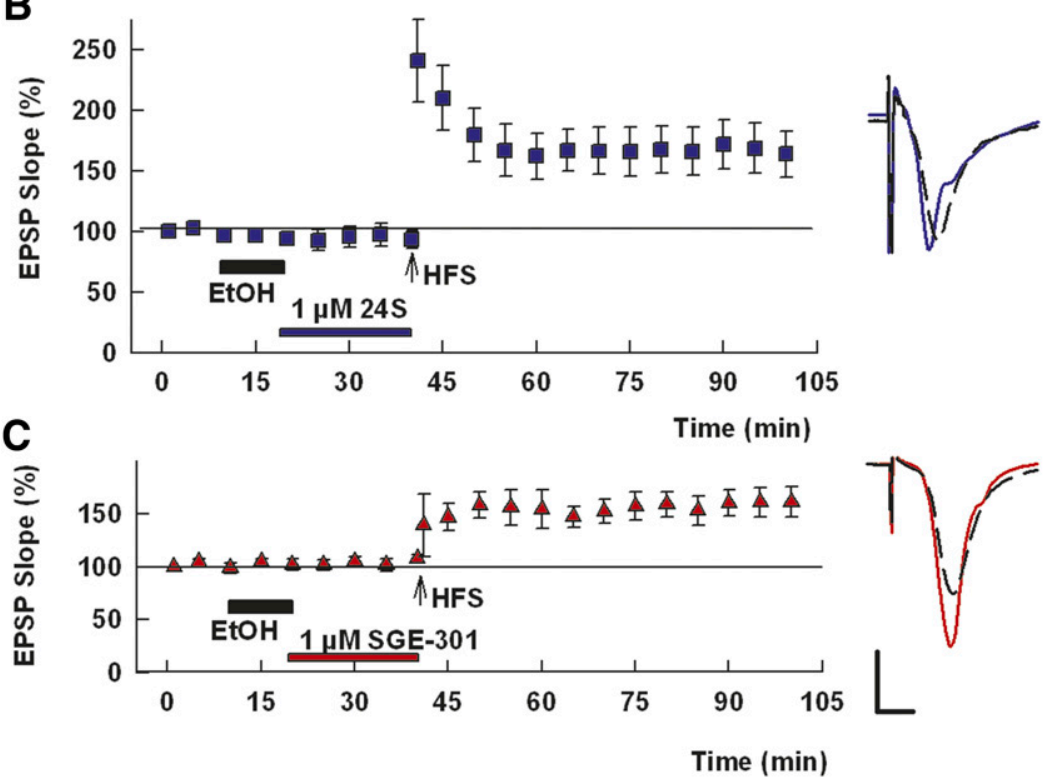

Fig. 3. 24S-HC and SGE-301 both reverse the persisting effects of ethanol on LTP. (A) The graph shows the block of LTP in slices treated with $60 \mathrm{mM}$ ethanol for 15 minutes and then washed out for 30 minutes prior to delivery of HFS (arrow) (blue squares). 24S-HC $(1 \mu \mathrm{M})$ overcame the effects of ethanol and allowed robust LTP (gray circles). (B) 24S$\mathrm{HC}$ also overcame the persisting effects of ethanol on LTP when administered after ethanol. (C) A synthetic mimic of 24S-HC, SGE-301 $(1 \mu \mathrm{M})$, also overcame the persisting effects of ethanol on LTP. Traces show representative fEPSPs as in Fig. 2 with solid traces taken 60 minutes after HFS. In (A), the upper set of traces is with 24S-HC. Calibration: $1 \mathrm{mV}, 5$ milliseconds. 


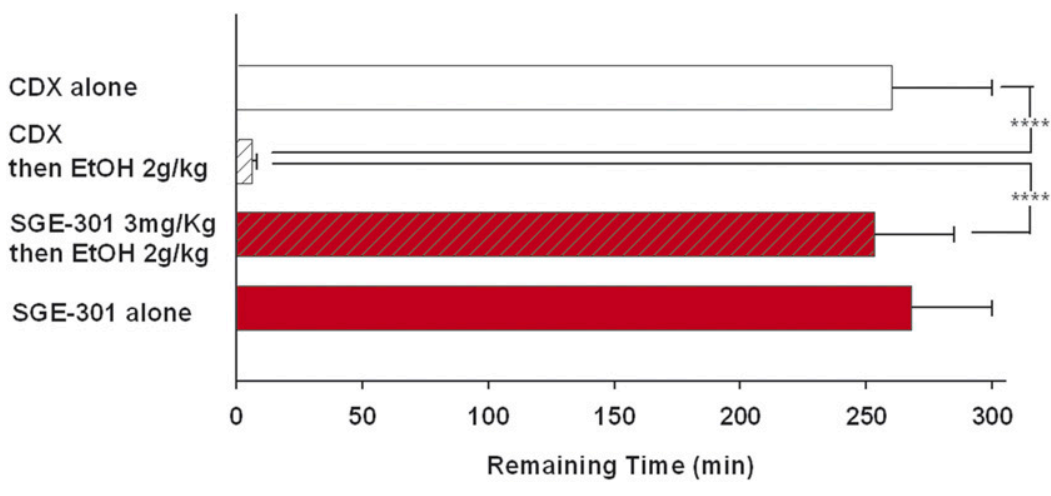

Fig. 4. SGE-301 overcomes the effects of ethanol on onetrial inhibitory avoidance learning. The top bar depicts the latency for rats to enter the dark chamber in the absence of drug treatment (saline controls) 24 hours after receiving a shock upon entry into the dark compartment during conditioning. All of these animals learned the task and failed to enter the dark chamber during the 300-second test. Rats treated with $2 \mathrm{~g} / \mathrm{kg}$ ethanol 15 minutes prior to the conditioning trial showed a marked decrement in learning and readily entered the dark chamber. Pretreatment with SGE-301 prevented the acute effects of ethanol on learning compared with controls pretreated with vehicle alone (CDX) prior to ethanol. SGE-301 alone had no effect on learning compared with vehicle controls. $* * * * P \leq 0.0001$ by one-way ANOVA, followed by Dunnett's multiple comparisons test vs. ethanol $(\mathrm{F}=17.60)$. on hippocampal NMDARs, synaptic plasticity, and one-trial inhibitory avoidance learning (Izumi and Zorumski, 2020). With the exception of effects on the block of NMDARs by ethanol, these actions were mimicked by ISRIB, a selective inhibitor of the ISR (Sidrauski et al., 2013; Sekine et al., 2015; Costa-Mattioli and Walter, 2020; Izumi and Zorumski, 2020). The LXR agonist and ISRIB also overcame prolonged adverse effects of ethanol on LTP that are observed after ethanol washout. Prior studies have demonstrated that neuronal stress resulting from aging and NMDAR activation promotes translocation of CYP46A1, the enzyme responsible for $24 \mathrm{~S}-\mathrm{HC}$ synthesis (Russell et al., 2009), from ER to plasma membrane and $24 \mathrm{~S}-\mathrm{HC}$ synthesis resulting in neuronal survival (Sodero et al., 2011a,b, 2012). Other studies indicate that knockdown of CYP46A1 in the hippocampus activates ER stress responses, apoptotic neuronal damage, and cognitive dysfunction (Burlot et al., 2015; Djelti et al., 2015); related effects on neuronal function are observed in striatum (Boussicault et al., 2016) and cerebellum (Nobrega et al., 2019). These observations prompted us to examine whether the oxysterol $24 \mathrm{~S}-\mathrm{HC}$ shared the effects of GW3965, given that this oxysterol is an endogenous agonist at LXRs (Janowski et al., 1999; Sun et al., 2016b).

We found that $24 \mathrm{~S}-\mathrm{HC}$ and a synthetic oxysterol analog, SGE-301, shared certain effects of GW3965, overcoming acute block of NMDAR fEPSPs by ethanol and prolonged effects of ethanol on LTP after ethanol washout. SGE-301, an agent with good bioavailability and brain penetration after systemic administration (Paul et al., 2013), also reversed acute effects of ethanol on one-trial learning. In contrast, the oxysterols had no effect on the acute direct inhibition of LTD by high concentrations of ethanol, differing from the LXR agonist and ISR inhibitor. Unexpectedly, we found that 24S-HC had no effect on acute block of LTP by ethanol (Fig. 2C), whereas SGE-301 overcame this effect of ethanol (Fig. 2D). However, both oxysterols overcame the persistent LTP inhibition that is observed after ethanol washout, including when the oxysterols were administered after ethanol (Fig. 3). We do not presently understand reasons for the differences in 24S-HC and SGE301 on acute LTP inhibition. Although we evaluated $24 \mathrm{~S}-\mathrm{HC}$ over a 10-fold range of concentrations to account for differences in potency, we note that SGE-301 was engineered with a $3 \alpha$-methyl group that dampens its metabolism and may alter its plasma membrane and intracellular distribution (Paul et al., 2013; Chen et al., 2018). The present studies were also done in juvenile male rats, consistent with our prior studies of ethanol (Izumi et al., 2005b, 2007; Izumi and Zorumski, 2020), but we have not observed differences between male and female rodents in oxysterol levels or responses in other studies (Popiolek et al., 2020).

Our results demonstrate that the oxysterols mimic only certain effects of the LXR agonist and the ISR inhibitor, indicating that the oxysterols clearly differ from both of these agents. Consistent with these observations, prior work indicates that SGE-301 has no clear effects on LXR, unlike 24SHC (Paul et al., 2013). In contrast, both SGE-301 and 24S-HC are potent and effective NMDAR PAMs (Paul et al., 2013), raising the strong likelihood that effects on NMDARs may primarily be responsible for the common constellation of oxysterol effects we have observed. Based on studies to date, NMDAR enhancement by the oxysterols is not shared by either GW3965 or ISRIB (Izumi and Zorumski, 2020).

In prior studies, we showed that the effects of a high concentration of ethanol on LTP and LTD likely result from different mechanisms. Effects on NMDARs and LTD mimic the effects of selective antagonists of NMDARs that express GluN2B subunits and reverse readily after ethanol washout. Effects on LTP are more complex and persistent and involve a form of metaplasticity after untimely NMDAR activation during the period of ethanol exposure (Izumi et al., 2005b; Zorumski et al., 2014). As a result, complete NMDAR inhibition with 2-amino-5-phosphonovalerate during ethanol exposure prevents persisting effects on LTP (Tokuda et al., 2011). In the present study, we found that the persisting effects of ethanol on LTP were overcome by both oxysterols, but, contrary to expectations, neither oxysterol altered the acute block of LTD, despite overcoming the block of hippocampal NMDARs by ethanol. The latter observations raise the possibility that more complex intracellular and perhaps intercellular signaling contributes to ethanol-mediated block of LTD. As one possibility, ISR inhibition overcomes ethanolmediated LTD inhibition, despite having no effect on the block of NMDARs (Izumi and Zorumski, 2020). Prior studies indicate that activation of ER stress promotes synthesis of endogenous neurosteroids (pregnenolone) from cholesterol (Barbero-Camps et al., 2014), and we previously found that induction of homosynaptic LTD in the CA1 region involves local NMDAR-dependent synthesis of GABAergic $5 \alpha$-reduced neurosteroids, downstream products of pregnenolone (Izumi et al., 2013). NMDAR-dependent LTD induction is also accompanied by metaplastic LTP inhibition involving neurosteroids (Izumi et al., 2013), as is LTP inhibition by pharmacological NMDAR activation (Izumi et al., 2007; Tokuda et al., 2011). These observations raise a possible role for ER stress 
and ISR-mediated intracellular signaling in determining effects of a variety of stressors on LTD and LTP (BarberoCamps et al., 2014; DiPrisco et al., 2014; Costa-Mattioli and Walter, 2020).

We also note that ethanol's ability to inhibit LTP via an NMDAR-dependent mechanism is shared with other noncompetitive NMDAR antagonists that also impair memory, most notably subanesthetic ketamine (Izumi and Zorumski, 2014). Both subanesthetic ketamine and ethanol appear to inhibit GluN2B-expressing NMDARs preferentially in the CA1 region at concentrations that inhibit LTP (Gerhard et al., 2020), and trigger NMDAR activation of unblocked NMDARs. Also, another synthetic oxysterol NMDAR PAM, SGE-201, overcomes persisting adverse effects of subanesthetic ketamine on LTP, and SGE-201 and SGE-301 reverse certain effects of related agents phencyclidine (PCP) and MK801 on cognition and behavior (Paul et al., 2013). In the latter studies, effects of oxysterols on PCP-induced changes were observed with subchronic PCP dosing and observed after drug washout. Some evidence also suggests that $24 \mathrm{~S}-\mathrm{HC}$ preferentially enhances tonic GluN2B-expressing NMDARs (Wei et al., 2019, but see Paul et al., 2013).

LXR agonists and ISR inhibitors have therapeutic potential as cognitive enhancers in neuropsychiatric illnesses (Wang et al., 2002; Donkin et al., 2010; Baez-Becerra et al., 2018). LXR agonists also have anti-inflammatory and neuroprotective effects that contribute to their CNS actions (Mouzat et al., 2019) but can produce significant side effects via changes in lipid metabolism and the importance of stress mechanisms for survival and function of cells and circuits (Wang and Tontonoz, 2018). The oxysterols offer an alternative approach based on their prominent effects as broad spectrum NMDAR PAMs without significant effects on other glutamate or $\mathrm{GABA}_{\mathrm{A}}$ receptors (Paul et al., 2013). With NMDAR enhancement, however, comes potential risks of excitotoxicity, although other studies have found both enhanced (Yamanaka et al., 2011, 2014; Sun et al., 2017) and diminished (Okabe et al., 2013; Ishikawa et al., 2016) neurotoxicity resulting from these agents. It is also intriguing that an oxysterol NMDAR PAM appears to be well tolerated in humans and is now in early testing as a possible cognitive enhancer (Koenig et al., 2019). NMDAR-mediated metaplasticity contributes to defects in LTP and learning in other conditions of neuronal stress; thus, effects observed here could be relevant for other neuropsychiatric illnesses (Zorumski and Izumi, 2012).

It is interesting and surprising that neither oxysterol overcame the acute effects of ethanol on LTD and only SGE-301 overcame acute LTP inhibition; the latter effect may reflect a difference in potency between the oxysterols. SGE-301 also overcame effects on one-trial avoidance learning, a form of learning linked to hippocampal LTP (Whitlock et al., 2006). However, both oxysterols overcame more persistent LTP inhibition after ethanol washout, including reversal of LTP inhibition when the oxysterols were administered after ethanol removal. We did not systematically explore how long after ethanol the oxysterols could be administered, but the combination of ex vivo and in vivo results suggests that persisting changes in synaptic plasticity likely account for at least some of ethanol's effects on cognition during and after bouts of intoxication. Prior stu(Parada et al., 2011)dies have observed persisting cognitive changes, including learning defects after acute ethanol intoxication, resulting in altered cognitive performance during alcohol hangovers (Crews et al., 2004; Silvestre de Ferron et al., 2015; Gunn et al., 2018; West et al., 2018; Contreras et al., 2019) and perhaps contributing to longerterm impairment from ethanol.

\section{Acknowledgments}

The authors thank Kazuko Izumi for technical assistance.

\section{Authorship Contributions}

Participated in research design: Izumi, Mennerick, Zorumski. Conducted experiments: Izumi.

Contributed new reagents or analytic tools: Doherty. Performed data analysis: Izumi, Zorumski.

Wrote or contributed to the writing of the manuscript: Izumi, Mennerick, Doherty, Zorumski.

\section{References}

Abrahao KP, Salinas AG, and Lovinger DM (2017) Alcohol and the brain: neuronal molecular targets, synapses, and circuits. Neuron 96:1223-1238.

Báez-Becerra C, Filipello F, Sandoval-Hernández A, Arboleda H, and Arboleda G (2018) Liver X receptor agonist GW3965 regulates synaptic function upon amyloid beta exposure in hippocampal neurons. Neurotox Res 33:569-579.

Barbero-Camps E, Fernández A, Baulies A, Martinez L, Fernández-Checa JC, and Colell A (2014) Endoplasmic reticulum stress mediates amyloid $\beta$ neurotoxicity via mitochondrial cholesterol trafficking. Am J Pathol 184:2066-2081.

Boussicault L, Alves S, Lamazière A, Planques A, Heck N, Moumné L, Despres G, Bolte S, Hu A, Pagès C, et al. (2016) CYP46A1, the rate-limiting enzyme for cholesterol degradation, is neuroprotective in Huntington's disease. Brain 139: 953-970.

Burlot M-A, Braudeau J, Michaelsen-Preusse K, Potier B, Ayciriex S, Varin J, Gautier B, Djelti F, Audrain M, Dauphinot L, et al. (2015) Cholesterol 24hydroxylase defect is implicated in memory impairments associated with Alzheimer-like Tau pathology. Hum Mol Genet 24:5965-5976.

Chen DM, Ziolkowski L, Benz A, Qian M, Zorumski CF, Covey DF, and Mennerick S (2018) A clickable oxysterol photolabel retains NMDA receptor activity and accumulates in neurons. Front Neurosci 12:923

Chen G, Ma C, Bower KA, Shi X, Ke Z, and Luo J (2008) Ethanol promotes endoplasmic reticulum stress-induced neuronal death: involvement of oxidative stress. $J$ Neurosci Res 86:937-946.

Contreras A, Morales L, and Del Olmos N (2019) The intermittent administration of ethanol during the juvenile period produces changes in the expression of hippocampal genes and proteins and deterioration of spatial memory. Behav Brain Res 372:112033.

Costa-Mattioli M and Walter P (2020) The integrated stress response: from mechanism to disease. Science 368:eaat5314.

Crews FT, Collins MA, Dlugos C, Littleton J, Wilkins L, Neafsey EJ, Pentney R, Snell LD, Tabakoff B, Zou J, et al. (2004) Alcohol-induced neurodegeneration: when, where and why? Alcohol Clin Exp Res 28:350-364.

Di Prisco GV, Huang W, Buffington SA, Hsu C-C, Bonnen PE, Placzek AN, Sidrauski C, Krnjević K, Kaufman RJ, Walter P, et al. (2014) Translational control of mGluRdependent long-term depression and object-place learning by eIF2 $\alpha$. Nat Neurosci 17:1073-1082.

Djelti F, Braudeau J, Hudry E, Dhenain M, Varin J, Bièche I, Marquer C, Chali F, Ayciriex S, Auzeil N, et al. (2015) CYP46A1 inhibition, brain cholesterol accumulation and neurodegeneration pave the way for Alzheimer's disease. Brain 138: $2383-2398$.

Donkin JJ, Stukas S, Hirsch-Reinshagen V, Namjoshi D, Wilkinson A, May S, Chan J, Fan J, Collins J, and Wellington CL (2010) ATP-binding cassette transporter A1 mediates the beneficial effects of the liver X receptor agonist GW3965 on object recognition memory and amyloid burden in amyloid precursor protein/presenilin 1 mice. J Biol Chem 285:34144-34154.

Gerhard DM, Pothula S, Liu R-J, Wu M, Li X-Y, Girgenti MJ, Taylor SR, Duman CH, Delpire E, Picciotto M, et al. (2020) GABA interneurons are the cellular trigger for ketamine's rapid antidepressant actions. J Clin Invest 130:1336-1349.

Gunn C, Mackus M, Griffin C, Munafô MR, and Adams S (2018) A systematic review of the next-day effects of heavy alcohol consumption on cognitive performance. Addiction 113:2182-2193.

Ishikawa M, Yoshitomi T, Zorumski CF, and Izumi Y (2016) 24(S)-Hydroxycholesterol protects the ex vivo rat retina from injury by elevated hydrostatic pressure. Sci Rep 6:33886.

Izumi Y, Auberson YP, and Zorumski CF (2006) Zinc modulates bidirectional hippocampal plasticity by effects on NMDA receptors. $J$ Neurosci 26:7181-7188.

Izumi Y, Kitabayashi R, Funatsu M, Izumi M, Yuede C, Hartman RE, Wozniak DF, and Zorumski CF (2005a) A single day of ethanol exposure during development has persistent effects on bi-directional plasticity, N-methyl-D-aspartate receptor function and ethanol sensitivity. Neuroscience 136:269-279.

Izumi Y, Murayama K, Tokuda K, Krishnan K, Covey DF, and Zorumski CF (2007) GABAergic neurosteroids mediate the effects of ethanol on long-term potentiation in rat hippocampal slices. Eur J Neurosci 26:1881-1888.

Izumi Y, Nagashima K, Murayama K, and Zorumski CF (2005b) Acute effects of ethanol on hippocampal long-term potentiation and long-term depression are mediated by different mechanisms. Neuroscience 136:509-517.

Izumi Y, O'Dell KA, and Zorumski CF (2013) Metaplastic LTP inhibition after LTD induction in CA1 hippocampal slices involves NMDA Receptor-mediated Neurosteroidogenesis. Physiol Rep 1:e00133. 
Izumi Y and Zorumski CF (2014) Metaplastic effects of subanesthetic ketamine on CA1 hippocampal function. Neuropharmacology 86:273-281.

Izumi Y and Zorumski CF (2020) Inhibitors of cellular stress overcome acute effects of ethanol on hippocampal plasticity and learning. Neurobiol Dis 141:104875.

Janowski BA, Grogan MJ, Jones SA, Wisely GB, Kliewer SA, Corey EJ, and Mangelsdorf DJ (1999) Structural requirements of ligands for the oxysterol liver X receptors LXRalpha and LXRbeta. Proc Natl Acad Sci USA 96:266-271.

Koenig H, Murck H, Luo Y, Webster I, Quirk M, Kanes S, and Doherty J (2019) M143. Cognitive performance after repeated administration of the NMDA positive allosteric modulator SAGE-718 in healthy volunteers. ACNP 58th Annual Meeting Poster Session 1. Neuropsychopharmacol 44:78-229.

Linsenbardt AJ, Taylor A, Emnett CM, Doherty JJ, Krishnan K, Covey DF, Paul SM Zorumski CF, and Mennerick S (2014) Different oxysterols have opposing actions at N-methyl-D-aspartate receptors. Neuropharmacology 85:232-242.

Magne L, Blanc E, Legrand B, Lucas D, Barouki R, Rouach H, and Garlatti M (2011) ATF4 and the integrated stress response are induced by ethanol and cytochrome P450 2E1 in human hepatocytes. J Hepatol 54:729-737.

Mouzat K, Chudinova A, Polge A, Kantar J, Camu W, Raoul C, and Lumbroso S (2019) Regulation of brain cholesterol: what role do liver X receptors play in neurodegenerative diseases? Int J Mol Sci 20:3858.

Nóbrega C, Mendonça L, Marcelo A, Lamazière A, Tomé S, Despres G, Matos CA, Mechmet F, Langui D, den Dunnen W, et al. (2019) Restoring brain cholesterol turnover improves autophagy and has therapeutic potential in mouse models of spinocerebellar ataxia. Acta Neuropathol 138:837-858.

Okabe A, Urano Y, Itoh S, Suda N, Kotani R, Nishimura Y, Saito Y, and Noguchi N (2013) Adaptive responses induced by 24S-hydroxycholesterol through liver X receptor pathway reduce 7-ketocholesterol-caused neuronal cell death. Redox Biol 2 28-35.

Oslin DW and Cary MS (2003) Alcohol-related dementia: validation of diagnostic criteria. Am J Geriatr Psychiatry 11:441-447.

Parada M, Corral M, Caamaño-Isorna F, Mota N, Crego A, Holguín SR, and Cadaveira F (2011) Binge drinking and declarative memory in university students. Alcohol Clin Exp Res 35:1475-1484.

Paul SM, Doherty JJ, Robichaud AJ, Belfort GM, Chow BY, Hammond RS, Crawford DC, Linsenbardt AJ, Shu H-J, Izumi Y, et al. (2013) The major brain cholesterol metabolite 24(S)-hydroxycholesterol is a potent allosteric modulator of N-methylD-aspartate receptors. J Neurosci 33:17290-17300.

Popiolek M, Izumi Y, Hopper AT, Dai J, Miller S, Shu H-J, Zorumski CF, and Mennerick S (2020) Effects of CYP46A1 inhibition on long-term-depression in hippocampal slices ex vivo and 24S-hydroxycholesterol levels in mice in vivo. Front Mol Neurosci 13:568641.

Rong X, Albert CJ, Hong C, Duerr MA, Chamberlain BT, Tarling EJ, Ito A, Gao J, Wang B, Edwards PA, et al. (2013) LXRs regulate ER stress and inflammation through dynamic modulation of membrane phospholipid composition. Cell Metab 18:685-697.

Russell DW, Halford RW, Ramirez DMO, Shah R, and Kotti T (2009) Cholesterol 24hydroxylase: an enzyme of cholesterol turnover in the brain. Annu Rev Biochem $\mathbf{7 8}$ $1017-1040$.

Sekine Y, Zyryanova A, Crespillo-Casado A, Fischer PM, Harding HP, and Ron D (2015) Stress responses. Mutations in a translation initiation factor identify the target of a memory-enhancing compound. Science 348:1027-1030.

Sidrauski C, Acosta-Alvear D, Khoutorsky A, Vedantham P, Hearn BR, Li H, Gamache K, Gallagher CM, Ang KK, Wilson C, et al. (2013) Pharmacological brakerelease of mRNA translation enhances cognitive memory. eLife 2:e00498.

Silvestre de Ferron B, Bennouar KE, Kervern M, Alaux-Cantin S, Robert A, Rabiant K, Antol J, Naassila M, and Pierrefiche O (2015) Two binges of ethanol a day keep the memory away in adolescent rats: key role for GluN2B subunit. Int $J$ Neuropsychopharmacol 19:pyv087.
Sodero AO, Trovò L, Iannilli F, Van Veldhoven P, Dotti CG, and Martin MG (2011a) Regulation of tyrosine kinase B activity by the Cyp46/cholesterol loss pathway in mature hippocampal neurons: relevance for neuronal survival under stress and in aging. $J$ Neurochem 116:747-755.

Sodero AO, Vriens J, Ghosh D, Stegner D, Brachet A, Pallotto M, Sassoè-Pognetto M, Brouwers JF, Helms JB, Nieswandt B, et al. (2012) Cholesterol loss during glutamate-mediated excitotoxicity. EMBO J 31:1764-1773.

Sodero AO, Weissmann C, Ledesma MD, and Dotti CG (2011b) Cellular stress from excitatory neurotransmission contributes to cholesterol loss in hippocampal neurons aging in vitro. Neurobiol Aging 32:1043-1053.

Sun M-Y, Izumi Y, Benz A, Zorumski CF, and Mennerick S (2016a) Endogenous 24Shydroxycholesterol modulates NMDAR-mediated function in hippocampal slices. $J$ Neurophysiol 115:1263-1272.

Sun M-Y, Linsenbardt AJ, Emnett CM, Eisenman LN, Izumi Y, Zorumski CF, and Mennerick S (2016b) 24(S)-Hydroxycholesterol as a modulator of neuronal signaling and survival. Neuroscientist 22:132-144.

Sun M-Y, Taylor A, Zorumski CF, and Mennerick SJ (2017) 24S-Hydroxycholesterol and 25-hydroxycholesterol differentially impact hippocampal neuronal survival following oxygen-glucose deprivation. PLoS One 12:e174416.

Tokuda K, Izumi Y, and Zorumski CF (2011) Ethanol enhances neurosteroidogenesis in hippocampal pyramidal neurons by paradoxical NMDA receptor activation. $J$ Neurosci 31:9905-9909.

Tokuda K, O'Dell KA, Izumi Y, and Zorumski CF (2010) Midazolam inhibits hippocampal long-term potentiation and learning through dual central and periphera benzodiazepine receptor activation and neurosteroidogenesis. $J$ Neurosci 30 : 16788-16795.

Wang B and Tontonoz P (2018) Liver X receptors in lipid signalling and membrane homeostasis. Nat Rev Endocrinol 14:452-463.

Wang L, Schuster GU, Hultenby K, Zhang Q, Andersson S, and Gustafsson J-A (2002) Liver X receptors in the central nervous system: from lipid homeostasis to neuronal degeneration. Proc Natl Acad Sci USA 99:13878-13883.

Wei X, Nishi T, Kondou S, Kimura H, and Mody I (2019) Preferential enhancement of GluN2B-containing native NMDA receptors by the endogenous modulator 24Shydroxycholesterol in hippocampal neurons. Neuropharmacology 148:11-20.

West RK, Maynard ME, and Leasure JL (2018) Binge ethanol effects on prefrontal cortex neurons, spatial working memory and task-induced neuronal activation in male and female rats. Physiol Behav 188:79-85.

Whitlock JR, Heynen AJ, Shuler MG, and Bear MF (2006) Learning induces longterm potentiation in the hippocampus. Science 313:1093-1097.

White AM (2003) What happened? Alcohol, memory blackouts, and the brain. Alcohol Res Health 27:186-196.

Yamanaka K, Saito Y, Yamamori T, Urano Y, and Noguchi N (2011) 24(S)-hydroxycholesterol induces neuronal cell death through necroptosis, a form of programmed necrosis. J Biol Chem 286:24666-24673.

Yamanaka K, Urano Y, Takabe W, Saito Y, and Noguchi N (2014) Induction of apoptosis and necroptosis by 24(S)-hydroxycholesterol is dependent on activity of acyl-CoA:cholesterol acyltransferase 1. Cell Death Dis 5:e990.

Zorumski CF and Izumi Y (2012) NMDA receptors and metaplasticity: mechanisms and possible roles in neuropsychiatric disorders. Neurosci Biobehav Rev 36: 989-1000.

Zorumski CF, Mennerick S, and Izumi Y (2014) Acute and chronic effects of ethanol on learning-related synaptic plasticity. Alcohol 48:1-17.

Address correspondence to: Charles F. Zorumski, Department of Psychiatry, Washington University School of Medicine, 660 South Euclid Ave., St. Louis, MO 63110. E-mail: zorumskc@wustl.edu 